\section{Occurrence and severity of upper eyelid skin contracture in facial nerve palsy}

K Ziahosseini' ${ }^{1}$ V Venables², C Neville², C Nduka ${ }^{3}$, B Patel ${ }^{4}$ and R Malhotra ${ }^{1}$

\begin{abstract}
Purpose To describe the occurrence and severity of upper eyelid skin contracture in facial nerve palsy (FNP).

Methods We enroled consecutive patients with unilateral FNP into this study. Patients with previous upper eyelid surgery for either side were excluded. We developed a standardised technique to measure the distance between the upper eyelid margin and the lower border of brow (LMBD). FNP was graded using the Sunnybrook grading scale. Its aetiology, duration, and treatment were noted. Upper and lower marginal reflex distance and lagophthalmos were also noted. Results Sixty-six patients (mean age 51 years) were included. FNP was owing to a variety of aetiologies. LMBD on the paralytic side was shorter than the normal contralateral side in $47(71 \%)$, equal in $15(23 \%)$, and larger in four $(6 \%)$ patients. The mean contracture was $3.4 \mathrm{~mm}$ (median: 3, range: 1-12) with 11 (17\%) patients showing $5 \mathrm{~mm}$ or more of skin contracture. The mean LMBD on the paralytic side in all patients was significantly smaller than the contralateral side; $30 \pm 3.7$ (median: 30 ; 95\% CI 29-31) compared with $32 \pm 3.7$ (median: 32; 95\% CI 32-33), respectively, $\boldsymbol{P}<\mathbf{0 . 0 0 0 1}$, two-tailed paired $\boldsymbol{t}$-test.

Conclusion To our knowledge, this is the first study that quantitatively demonstrates contraction of the upper eyelid skin in FNP. This finding is valuable in directing optimal early management to minimise skin contracture and to caution surgeons against unnecessary upper eyelid skin excision.

Eye (2016) 30, 713-717; doi:10.1038/eye.2016.21; published online 4 March 2016
\end{abstract}

\section{Introduction}

Facial nerve palsy (FNP) is a disfiguring condition with a range of aetiologies and prognosis that causes severe functional and psychological disability. Clinicians and facial therapists, who provide care for patients with FNP, are anecdotally aware of the atrophic appearance of the upper- and lower-eyelid skin and shortening that can occur of the eyelid skin. Aramideh et $a l^{1}$ described the phenomenon of 'thixothropy' of the levator muscle in facial paralysis. Thixothropy is stiffness of a striated muscle because of the formation of tight crossbridges between actin and myosin filaments within muscle fibres, leading to shortening of the sarcomere. They showed a moderate-to-significant reduction of lagophthalmos after manual stretch of upper eyelid. It is recognised that local atrophy of muscle, bone, and/or soft tissue can result from disuse or diminished activity. Furthermore, muscle-pump paralysis also reduces venous tone and therefore may raise the hydrostatic pressure within tissue. These can lead to fibrosis of the subcutis and trophic skin changes. ${ }^{2}$

There is currently no quantitative evidence of upper eyelid skin shrinkage in patients with facial nerve paralysis in the literature. We report the occurrence and severity of this complication and explore its correlation in this paper. The results of this study may be valuable in identification and utilisation of measures to prevent or reduce this complication and improve the management of FNP.

\section{Materials and methods}

We carried out a cross-sectional study of consecutive patients with unilateral FNP presenting to the tertiary referral centre facial palsy clinics at the Queen Victoria Hospital, East Grinstead, UK (December 2013-April 2014) and Moran Eye Centre, University of Utah, Salt Lake City, UT, USA (November 2014-April 2015). Institutional review board approval for this study was obtained. The study was conducted in

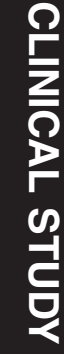

${ }^{1}$ Corneoplastic Unit, Queen Victoria Hospital, East Grinstead, UK

${ }^{2}$ Department of Therapy, Queen Victoria Hospital, East Grinstead, UK

${ }^{3}$ Department of Plastic Surgery, Queen Victoria Hospital, East Grinstead, UK

${ }^{4}$ Moran Eye Centre, University of Utah, Salt Lake City, UT, USA

Correspondence:

Current address:

K Ziahosseini,

Ophthalmology

Department, Norfolk and Norwich University Hospital, Norwich NR4 7UY, UK Tel: +44 (0)1603 288372; Fax: +44 (0)1603 288261. E-mail: kim.z@doctors.org.uk

Received: 21 July 2015 Accepted in revised form: 25 December 2015 Published online: 4 March 2016

This study was presented at The American Academy of Ophthalmology Annual Meeting, October 2014, and at The American Society of Ophthalmic Plastic and Reconstructive Surgery, Fall Scientific Symposium, October 2014. 
full accord with the tenets of the Declaration of Helsinki. Patients with bilateral FNP and previous upper eyelid surgery for either side were excluded. The aetiology, duration, and treatment of FNP were noted. Upper and lower marginal reflex distance (MRD) and lagophthalmos on gentle closure were measured in millimetres from standard photographs. Facial paralysis was graded using the Sunnybrook grading scale (SFGS). ${ }^{3}$

The SFGS composite score (complete paralysis $=0$, normal $=100$ ) has three components of resting symmetry (RS), voluntary movement (VM) and synkinesis (SYNK). The RS score ( 0 is normal, 4 is poor) compares the resting state of palpebral aperture, nasolabial fold and mouth to the normal contralateral side. The VM score evaluates the symmetry of brow elevation, gentle eye closure, and open mouth smile, snarl and lip pucker. It ranges between 5 (very poor score) to 25 (normal). The SYNK score ranges between 0 (no SYNK) to 15 (disfiguring SYNK). It rates the severity of SYNK on the five facial expressions used to assess VM.

We developed a standardised technique to measure the distance between the upper eyelid margin and the lower border of the brow hair in the mid-pupillary line, the 'lid margin to brow distance (LMBD)'. The assessor would ask the patient to look straight ahead. She/he would imagine a vertical line from the pupil to the brow, and would place their thumb on the brow where the two intersect. She/he would ask the patient to look down and while lifting the brow, would stretch the skin maximally so that all skin folds were smoothened. A paper ruler would then be held against the eyelid and the assessor would measure the vertical distance between the lash line and the base of the brow hair in millimetres. This was repeated on the contralateral side. Three assessors collected data. Each assessor measured both eyelids of every patient, using the technique described above, three times and averaged the results (Figure 1).

Upper and lower MRDs were calculated from standardised photographs by measuring the distance from the central pupil to the central upper- and lowereyelid margin and dividing this by the horizontal corneal diameter. This ratio was then multiplied by a constant $K=11.68$, derived from Hashemi et al. ${ }^{4}$

A total of 40 patients (40 in the paralysis group and 40 in the control group) were calculated to have $80 \%$ power to detect a difference between means of 1.74 with a significance level (alpha) of 0.05 (two-tailed). Data is given as mean \pm SD (95\% confidence interval).

Two-tailed paired $t$-test and Pearson's $r$ correlation were used when necessary. Graphpad prism 6 (Graphpad Software, La Jolla, CA, USA) was used for statistical analysis.
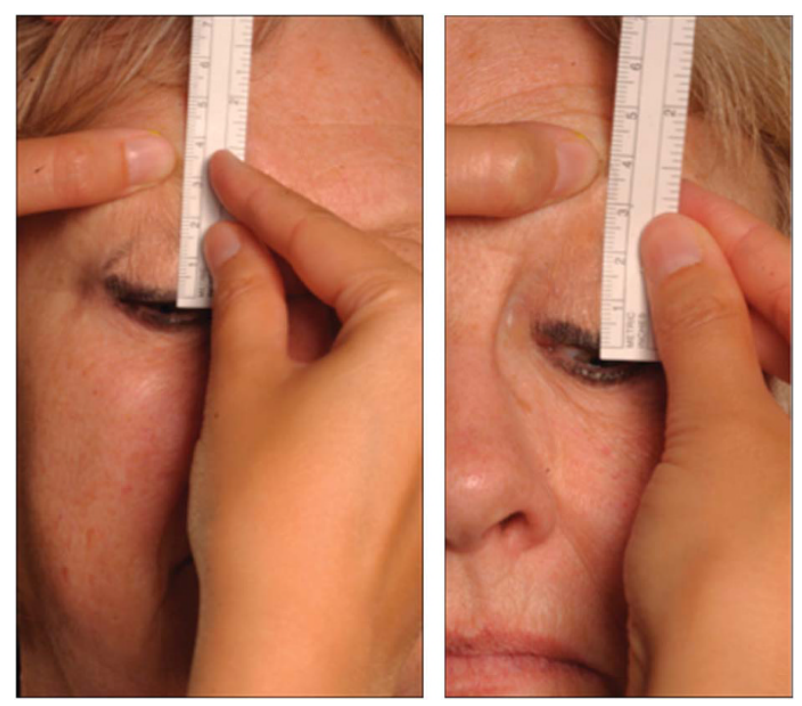

Figure 1 The technique of LMBD measurement in a patient with right Bell's palsy. Vertical skin striae show maximal skin stretch. Right LMBD: $30 \mathrm{~mm}$; left LMBD: $35 \mathrm{~mm}$.

\section{Results}

Sixty-six patients (mean age 51 years, median: 52, range: 7-79 years, 26 males and 40 females) were included in the study. FNP was due to a variety of aetiologies (32 Bell's palsy, 9 acoustic schwannoma, 8 parotid tumour, 7 trauma, 4 Ramsay Hunt Syndrome, 2 jugular paraganglioma, 1 Lyme disease, 1 lymphoma, 1 vasculitis, and 1 congenital). The mean duration of palsy was 60 months (median: 36 months, range: 2 months to 29 years).

LMBD on the affected side was shorter than the normal contralateral side in $47(71 \%)$, equal in $15(23 \%)$ and larger in four $(6 \%)$ patients. The mean contracture was $3.4 \mathrm{~mm}$ (median: 3, range: 1-12) with 11 (17\%) patients showing $5 \mathrm{~mm}$ or more of skin contracture. The mean LMBD on the paralytic side in all patients was significantly smaller than the contralateral side; $30 \pm 3.7$ (median: 30; 95\% CI 29-31) compared with $32 \pm 3.7$ (median: 32; 95\% CI 32-33), respectively, $P<0.0001$, two-tailed paired $t$-test.

Of the 32 patients with Bell's palsy, 62\% (20) showed upper eyelid skin contracture in comparison with $87 \%$ $(20 / 23)$ of patients with facial nerve resection in the context of surgery for acoustic schwannoma or parotid tumour, or trauma ( $P=0.04$, two-tailed, $\chi^{2}$-test). When present, however, the mean upper eyelid skin shortening was not significantly different between these 2 groups, $3.3 \mathrm{~mm}$ (median: 3 , range: 1-12) vs $3.4 \mathrm{~mm}$ (median: 3 , range: $1-8$ ).

The mean composite SFGS score was $42.5 \pm 21.9$ (95\% CI 37.1-48.0). The score for the three components (RS, VM, and SYNK) of SFGS, as well as the ocular components (RS eye score, VM eye closure) are given in Table 1. The data for upper and lower MRD and lagophthalmos on gentle closure are also given in Table 1. 
Table 1 Descriptive data

\begin{tabular}{|c|c|c|c|c|c|c|c|c|}
\hline & $\begin{array}{c}\text { Number of } \\
\text { values }\end{array}$ & Minimum & Maximum & Mean & Median & $\begin{array}{l}\text { Standard } \\
\text { deviation }\end{array}$ & $\begin{array}{c}\text { Lower } 95 \% \text { CI of } \\
\text { mean }\end{array}$ & $\begin{array}{c}\text { Upper } 95 \% \text { CI of } \\
\text { mean }\end{array}$ \\
\hline LMBD difference & 66 & -6 & 12 & 2.3 & 2.0 & 2.7 & 1.6 & 3.0 \\
\hline $\begin{array}{l}\text { Upper eyelid skin } \\
\text { shrinkage }\end{array}$ & 47 & 1 & 12 & 3.4 & 3.0 & 2.1 & 2.8 & 4.0 \\
\hline SFGS composite score & 66 & 0 & 83 & 42.5 & 45.0 & 21.9 & 37.1 & 48.0 \\
\hline RS score & 66 & 0 & 4 & 2.3 & 2.0 & 1.1 & 2.0 & 2.5 \\
\hline VM score & 66 & 5 & 24 & 14.6 & 15.0 & 4.8 & 13.4 & 15.8 \\
\hline SYNK score & 66 & 0 & 13 & 4.6 & 5.0 & 3.7 & 3.7 & 5.5 \\
\hline Upper MRD & 66 & 2 & 6.3 & 4.2 & 4.2 & 1.1 & 3.9 & 4.5 \\
\hline Lower MRD & 66 & 3.5 & 9.0 & 6.0 & 5.8 & 1.2 & 5.7 & 6.4 \\
\hline Lagophthalmos & 66 & 0 & 9 & 2.4 & 1.5 & 2.4 & 1.7 & 3.0 \\
\hline RS eye score & 66 & 0 & 1 & 0.9 & 1.0 & 0.4 & 0.8 & 0.9 \\
\hline VM eye closure & 66 & 1 & 5 & 3.7 & 4.0 & 1.2 & 3.4 & 4.0 \\
\hline
\end{tabular}

Abbreviations: MRD, marginal reflex distance; RS, resting symmetry; SFGS, Sunnybrook Facial Grading Scale; SYNK, synkinesis; VM, voluntary movement. Lagophthalmos on gentle closure and the ophthalmic component of resting symmetry score and voluntary movement scores.

Table 2 Correlation data between upper eyelid skin shrinkage and other variables

\begin{tabular}{lrc}
\hline & r-value & P-value \\
\hline Age & -0.09 & 0.4 \\
Duration (months) & -0.04 & 0.7 \\
SFGS composite score & -0.20 & 0.1 \\
RS score & 0.21 & 0.08 \\
VM score & -0.21 & 0.07 \\
SYNK score & -0.29 & $0.01^{*}$ \\
Upper MRD & 0.02 & 0.83 \\
Lower MRD & 0.33 & $0.006^{*}$ \\
Lagophthalmos & 0.32 & $0.007^{*}$ \\
RS eye score & 0.18 & 0.15 \\
VM eye closure & -0.26 & $0.031^{*}$ \\
\hline
\end{tabular}

Abbreviations: MRD, marginal reflex distance; RS, resting symmetry; SFGS, Sunnybrook Facial Grading Scale; SYNK, synkinesis; VM, voluntary movement. ${ }^{*} P<0.05$.

Pearson's $r$ correlation coefficients were computed to assess the association between upper eyelid skin shrinkage and variables such as age, duration of FNP, SFGS composite score and its components, upper and lower MRD and lagophthalmos (Table 2). We did not find a significant association with the duration of disease $(P=0.7)$. Indeed, upper eyelid skin shrinkage was recorded as early as 2-3 months from the onset of paralysis (Figure 2). There was also no significant correlation with age. We detected a low-grade positive association between the upper eyelid skin shrinkage and lower MRD ( $r=0.33, n=66, P=0.006)$. It was not surprising to detect a low-grade positive association between the upper eyelid shortening and lagophthalmos $(r=0.32, n=66, P=0.007)$. We also detected a low-grade negative correlation with the SYNK score $(r=-0.29$, $n=66, P=0.01$ ) suggesting that undesirable aberrant facial nerve regeneration and orbicularis SYNK may have a protective effect against eyelid skin contracture.

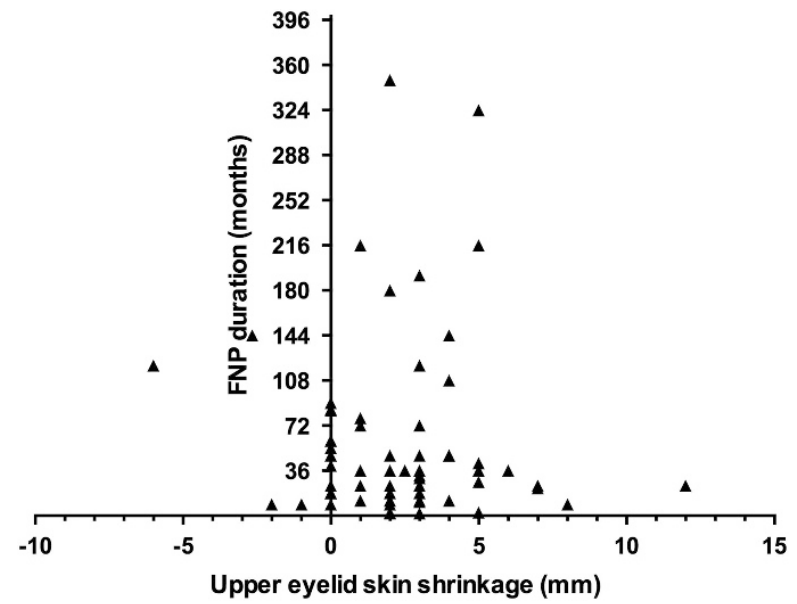

Figure 2 Scatter plot of duration of FNP and upper eyelid skin shortage.

\section{Discussion}

The phenomenon of skin and soft-tissue contraction secondary to a loss of constant or repetitive mechanical loading is familiar to many surgeons and is used in reverse when tissue expanders are used..$^{5}$ As well as being a routine phenomenon in abdominal and breast skin after pregnancy and breast-feeding, skin retraction is the mechanism by which skin redundancy is lost after liposuction. ${ }^{6}$ The other mechanisms of skin contracture is less well-studied and understood but has been reported in the field of hand surgery, where surgeons frequently battle with the consequences of loss of mobility on the availability of skin, as evidenced by the loss of wrinkles on the dorsum of the fingers after a period of immobilisation or fusion of an interphalangeal joint (Figure 3). ${ }^{7}$

To the best of our knowledge, this is the first study to attempt to quantify the occurrence and severity of upper 


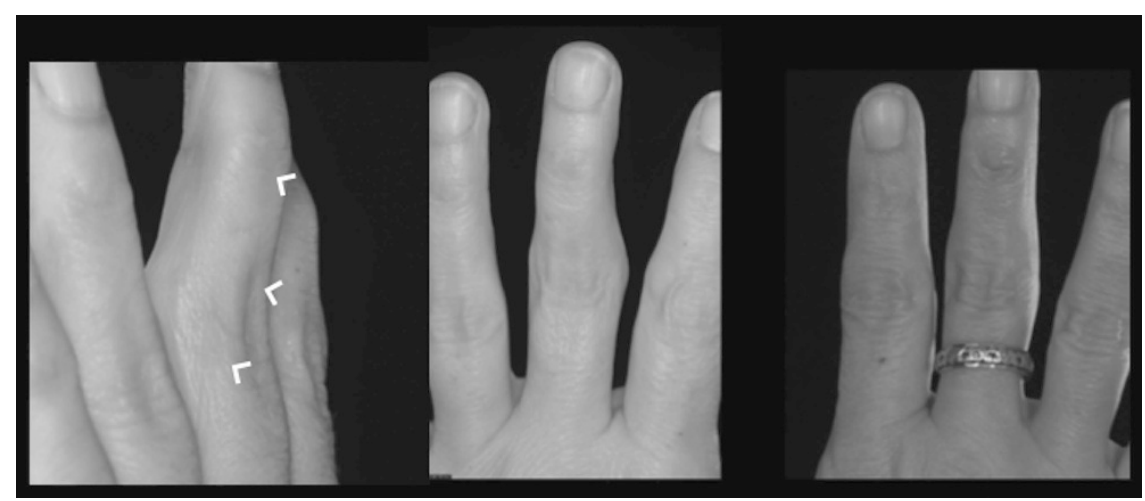

Figure 3 Left shows extension deformity of left middle finger. Left and centre show diminished wrinkles over the affected area (arrowheads) in comparison with normal right hand of the same patient.

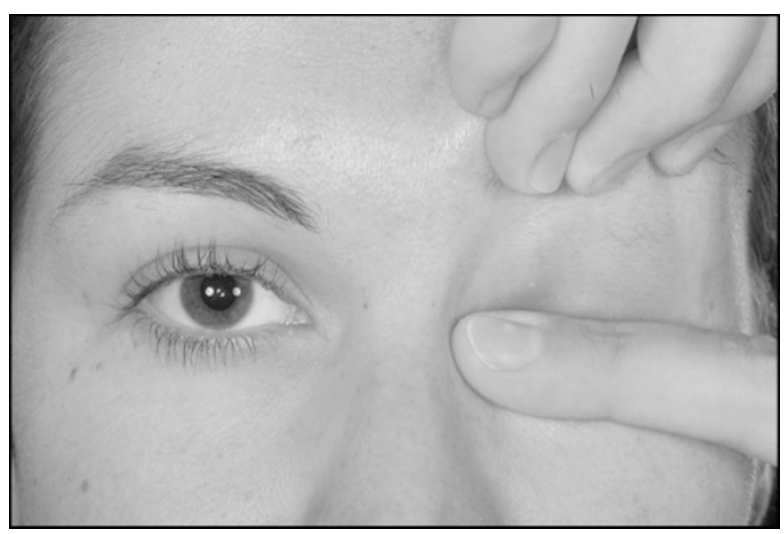

Figure 4 Shows manual stretch of left upper eyelid. Eyelid is held closed with a finger, whereas the eyebrow is stretched upward using the other hand. Patients are advised to maintain the stretch for $20 \mathrm{~s}$ and repeat three times a day.

lid skin contracture in FNP. Seventy-one per cent of patients in this cohort showed ipsilateral skin reduction and $17 \%$ showed $5 \mathrm{~mm}$ or more of skin shortening. The exact mechanism of this complication is difficult to explain and requires further study. Shortening of the levator muscle due to thixotropy may have a role in the early phase. This effect is partly reversible with manual stretch, which is widely recommended by facial therapists (Figure 4). It is possible that muscle and soft-tissue atrophy follows the denervation of the orbicularis oculi muscle as it occurs elsewhere in the body following denervation.

We did not detect any correlation between upper eyelid skin contracture and duration of paralysis. Indeed, some patients presented with it as early as $2-3$ months from the onset of paralysis. This is intriguing as one would naturally expect the skin shrinkage to progress with time. It may be due to heterogeneity of our patients. A longitudinal study is required to investigate this.

Upper eyelid skin contraction may be an important impediment to the optimum management of lagophthalmos. In the presence of skin contraction, upper eyelid loading with a standard range of weights between 1.0-1.4 g may not yield the desired, or expected improvement in lagophthalmos and may mislead the clinician into attempting a heavier weight with its potentially greater risk of prominence, poor contour, extrusion, and so on. ${ }^{8}$ Therefore, in our view the measurement of LMBD, which is simple and quick, should become part of the routine assessment of patients with facial paralysis. This will hopefully improve our understanding of this phenomenon.

Correction of lagophthalmos by lengthening the levator muscle has been recommended by some surgeons through the insertion of an interposition graft. In these cases a graft measuring double the vertical height of the measured lid retraction is interposed between the levator aponeurosis and the tarsal plate. ${ }^{9}$

Skin laxity and/or brow ptosis in older patients can mask the development of skin contraction. Our findings may have value in minimising skin excision in the management of young patients who are more concerned about their aesthetic outcome and keen to explore aesthetic rehabilitation with blepharoplasty. A brow lift, having verified that it will not result in lagophthalmos, would usually correct the functional and aesthetic problem ensued from pre-existing dermatochalsis that is exacerbated due to brow ptosis. If there is still significant skin excess (which would tend to be confined to the lateral third of the eyelid) a conservative skin reduction may be considered.

Our results showed a low-grade association between increasing upper eyelid skin contracture and lower eyelid retraction. Lower eyelid retraction is clearly multifactorial in FNP and is not an ideal measure of lower eyelid skin 
contraction. Further studies are required to assess lower eyelid skin contracture in FNP. We detected a low-grade negative association between SYNK score and skin contracture suggesting that aberrant facial nerve regeneration and orbicularis SYNK may have a protective effect against eyelid skin contracture. Upper eyelid skin shortening was also more prevalent in FNP due to facial nerve resection than Bell's palsy. This corresponds with the clinical experience of the authors that patients with dense paralysis often show more significant contracture and those with aberrant regeneration often show little contraction. Future studies on more homogeneous group of patients are required to establish the validity of these findings.

We use the SFGS as the preferred comprehensive grading scale in our institution. It evaluates symmetry at rest and during VMs and also scores SYNK to produce a single score. ${ }^{3}$ It is therefore a continuous scale that can be manipulated mathematically. It is arguably a more comprehensive and accurate description of facial nerve function than the more commonly known House Brackmann grading scale. It is reported to have better repeatability than the HBGS and has high inter-/intraobserver reliability when used either by novice and expert users. $^{10-12}$

In conclusion, this study is the first to show that significant, measurable skin contracture does occur in patients with FNP. Adding the simple measurement of LMBD to the routine assessment of these patients will add to our understanding of this phenomenon. Although this study included facial nerve weakness of varied aetiologies, future studies are needed to see if patients with complete transection of the facial nerve develop more severe contracture and at what rate. A longitudinal study would shed light on contracture over time in such patients. Once these findings are established, it would be fascinating to know if performing levator recession, with or without the insertion of gold weights, would reduce skin contracture. As one of the authors (BCKP) has treated several patients who have had skin resection performed during the insertion of gold weights and levator recession in the presence of dense facial palsies by the referring surgeons (they needed various procedures to improve the progressive lagophthalmos), based on the findings of this study, it would be prudent to minimise or avoid skin resection in such patients. Surgeons who manage patients with facial paralysis need to be aware of this phenomenon of skin contracture in patients with facial palsy.

\section{Summary}

What was known before

- Facial palsy is a disfiguring condition that causes severe functional and psychological disabilities.

- Management of ocular complication of facial palsy is challenging.

What this study adds

- Measurable upper eyelid skin shrinkage occurs in the majority of patients with facial palsy.

- Unnecessary upper eyelid skin excision should be avoided in these patients.

- Better understanding of this phenomenon will improve management of ocular surface exposure.

\section{Conflict of interest}

The authors declare no conflict of interest.

\section{References}

1 Aramideh M, Koelman JH, Devriese PP, VanderWerf F, Speelman JD. Thixotropy: a novel explanation for the cause of lagophthalmos after peripheral facial nerve palsy. $\mathrm{Br} J$ Ophthalmol 2002; 86(8): 839.

2 Trettin H. Neurologic principles of edema in inactivity. Z Lymphol 1992; 16: 14-16.

3 Ross BG, Fradet G, Nedzelski JM. Development of a sensitive clinical facial grading system. Otolaryngol Head Neck Surg 1996; 114: 380-386.

4 Hashemi H, Khabaz Khoob M, Yazdani K, Mehravaran S, Mohammad K, Fotouhi A. White- to-white corneal diameter in the Tehran Eye Study. Cornea 2010; 29: 9-12.

5 Wilhelmi BJ, Blackwell SJ, Mancoll JS, Phillips LG. Creep vs. stretch: a review of the viscoelastic properties of skin. Ann Plast Surg 1998; 41: 215-219.

6 Coleman WP IV, Hendry SL. Principles of liposuction. Semin Cutan Med Surg 2006; 25: 138-144.

7 Sprague BL. Proximal interphalangeal joint contractures and their treatment. J Trauma 1976; 16(4): 259-265.

8 Bladen JC, Norris JH, Malhotra R. Indications and outcomes for revision of gold weight implants in upper eyelid loading. Br J Ophthalmol 2012; 96: 485-489.

9 Guillou-Jamard MR, Labbé D, Bardot J, Benateau H. Paul Tessier's technique in the treatment of paralytic lagophthalmos by lengthening of the levator muscle: evaluation of 29 cases. Ann Plast Surg 2011; 67(6): S31-S35.

10 Kanerva M, Poussa T, Pitkaranta A. Sunnybrook and HouseBrackmann Facial Grading Systems: intrarater repeatability and interrater agreement. Otolaryngol Head Neck Surg 2006; 135: 865-871.

$11 \mathrm{Hu}$ WL, Ross B, Nedzelski J. Reliability of the Sunnybrook Facial Grading System by novice users. J Otolaryngol 2001; 30: 208-211.

12 Kayhan FT, Zurakowski D, Rauch SD. Toronto Facial Grading System: interobserver reliability. Otolaryngol Head Neck Surg 2000; 122: 212-215. 